\title{
Dieta de murciélagos filostómidos del valle de Kosñipata, San Pedro, Cusco - Perú
}

\author{
Diet of phyllostomid bats of Kosñipata Valley, San Pedro, Cusco - Peru
}

\begin{abstract}
1 Laboratorio de Palinología y Paleobotánica. Laboratorios de Investigación y Desarrollo (LID). Univerion y Desarrollo (LID). Universidad Peruana Cayetano Heredia. Av. Honorio Delgado 430, Urb. Ingeniería, S.M.P. Lima 31, Perú.

2 Laboratorio de Estudios en Biodiversidad Laboratorios de Investigación y Desarrollo (LID). Universidad Peruana Cayetano Heredia. Email Rossana Maguiña: nrmaguina@gmail.com
\end{abstract}

\author{
Rossana Maguiña1, Jessica Amanzo² y Luis Huamán ${ }^{1}$
}

\section{Resumen}

En el presente estudio se evaluó la dieta de murciélagos filostómidos de los bosques montanos del valle de Kosñipata (San Pedro, Cusco). Las especies evaluadas pertenecieron a los géneros Carollia, Sturnira y Anoura. Se analizó la dieta en base a la presencia de polen y semillas en muestras fecales. Se realizó un análisis de la importancia de las plantas consumidas, la amplitud del nicho alimenticio y el nivel de superposición del nicho alimenticio. Los resultados mostraron que la dieta de los murciélagos está compuesta en su mayoría por frutos y néctar-polen de las plantas. Los principales recursos registrados pertenecieron a los géneros Piper (registrado en $23,08 \%$ de las muestras), Cecropia (en $21,15 \%$ ) y Abutilon (en 13,46\%), los cuales fueron los recursos más importantes para la comunidad de murciélagos evaluada. La amplitud de nicho alimenticio para las 6 especies analizadas indicó que son especialistas en su dieta en el área de estudio. Por otro lado, los mayores niveles de superposición de nicho alimenticio ocurre entre las especies C. brevicauda, C. perspicillata y S. erythromos.

Palabras clave: Phyllostomidae; murciélago; frugívoro; nectarívoro; dieta.

\section{Abstract}

In this study had been evaluated the diet of phyllostomids bats of the montane forest of the Kosñipata Valley (San Pedro, Cusco). The species of bats evaluated belong to the genus Carollia, Sturnira and Anoura. The diet was evaluated regarding the presence of pollen and seeds in fecal samples. Also, it was analyzed the importance of the plants consumed, the food niche breath and the food niche overlap. The results showed that the diet of the bats is mostly compounded of fruit and nectar-pollen of the plants. The principal resources registered belong to the genus Piper (registered in $23,08 \%$ of the samples), Cecropia (in 21,15\%) and Abutilon (in $13,46 \%$ ), which was the most important resources for the community of bats evaluated. The food niche breath of the six species analyzed showed that they are specialist in their diet in the study zone. The highest level of food niche overlap occurs between the species C. brevicauda, C. perspicillata and S. erythromos.

Keywords: Phyllostomidae; bat; frugivorous; nectarivorous; diet.

\section{Introducción}

En el Perú, las investigaciones realizadas en el orden Chiroptera han servido para llenar vacíos de información sobre distribución, diversidad y sistemática de especies. Aunque la mayoría de ellos incluye aspectos ecológicos, son muy pocos los dedicados al estudio exclusivo de la dieta (Novoa et al. 2011, Arias et al. 2009, Gorchov et al. 1995, Ascorra et al. 1996).

La importancia de los estudios de dieta en murciélagos, específicamente en especies herbívoras, radica en los roles ecológicos de polinización y dispersión que cumplen dentro de su hábitat (Fleming 1988). Los murciélagos polinizadores son importantes en la fenología reproductiva y la estructura poblacional de las plantas, mientras que los dispersores se convierten en entes esenciales de la regeneración de bosques tropicales y del mantenimiento de la diversidad vegetal del mismo (Fleming 1988).

En el presente estudio, (1) se identifican los taxa componentes de la dieta del murciélagos frugívoros Carollia y Sturnira (Gardner 2007), y del género nectarívoro Anoura (Ortega y Alarcón 2007); y (2) se determinan los taxa más importantes en la dieta y realiza un análisis del nicho alimenticio, considerando la amplitud y superposición de nicho, con la finalidad de documentar las relaciones existentes entre los murciélagos y las plantas en el bosque nublado del valle de Kosñipata, San Pedro, Cusco.

\section{Material y métodos}

Área de estudio.- El estudio se realizó en el Valle de Kosñipata, en la localidad de San Pedro (Cusco), en un área circundante al alberge turístico "Cock of the Rocks". La zona se encuentra localizada en los bosques montano orientales del sureste amazónico, abarcando altitudes desde 1100 hasta $1600 \mathrm{~m}$. Comprende las siguientes zonas de vida según el sistema de clasificación de Holdrige (1978): Bosque muy Húmedo Montano Subtropical (bmh-MS), Bosque muy Húmedo Montano Bajo Subtropical (bmh-MBS) y Bosque Pluvial Montano Subtropical (bp-MS). El clima es templado, se ha reportado que dependiendo de la altura, el rango de temperatura varía entre 8 y $18{ }^{\circ} \mathrm{C}$, con una precipitación anual de 4510 a $5470 \mathrm{~mm}$ (SENAMHI 2010). La humedad relativa es alta durante todo el año, pero se observan dos épocas climáticas marcadas; la época de lluvias, que va de diciembre a abril, y la época seca, de mayo a noviembre, con escazas lluvias. Pacheco et al., (2004) describen la localidad de San Pedro como un área dominada por bosque secundario, con laderas moderadas de $20-30^{\circ}$, y varios riachuelos pequeños que alimentan un largo torrente con flujo hacia el este. Además mencionan que el paisaje está dominado por gruesas plantas de bambú trepador (Chusquea sp.), y por árboles cubiertos por epífitas. El acceso hacia esta zona se realiza desde la ciudad de Cusco, vía terrestre, por la carretera a Pilcopata.

Toma de datos.- Se llevó a cabo durante la época seca en julio del 2007 y la época de lluvias en marzo y abril del 2008. Las muestras fecales de los murciélagos proceden de los individuos capturados durante el estudio de Delgado (2010). Las muestras fueron conservadas en alcohol al 70\%. Las especies de murciélagos fueron determinadas usando claves taxonómicas especializadas (Gardner 2007, Angulo et al. 2004, Albuja 1999, 
Tabla 1. Frecuencia de los componentes registrados en las muestras fecales. Número de muestras $(N)$, polen $(P)$, semilla $(S)$, tejido vegetal $(T V)$, restos de insectos (I), muestra estéril (E).

\begin{tabular}{|c|c|c|c|c|c|c|c|}
\hline \multirow{2}{*}{ Especie } & \multirow{2}{*}{ Gremio alimenticio } & \multirow{2}{*}{$\mathbf{N}$} & \multicolumn{4}{|c|}{ Frecuencia de los componentes } & \multirow{2}{*}{ E } \\
\hline & & & $\mathbf{P}$ & 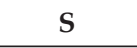 & TV & $\mathbf{I}$ & \\
\hline Carollia brevicauda & frugívoro & 10 & 6 & 1 & 9 & 1 & 0 \\
\hline Carollia perspicillata & frugívoro & 3 & 2 & 2 & 3 & 0 & 0 \\
\hline Carollia manu & frugívoro & 1 & 0 & 0 & 1 & 0 & 0 \\
\hline Sturnira erythromos & frugívoro & 9 & 8 & 3 & 5 & 0 & 1 \\
\hline Sturnira oporaphilum & frugívoro & 19 & 8 & 2 & 16 & 1 & 2 \\
\hline Sturnira tildae & frugívoro & 2 & 1 & 1 & 2 & 0 & 0 \\
\hline Sturnira lilium & frugívoro & 1 & 0 & 0 & 1 & 0 & 0 \\
\hline Anoura caudifer & nectarívoro & 6 & 5 & 0 & 4 & 0 & 0 \\
\hline Anoura geoffroyi & nectarívoro & 1 & 0 & 0 & 1 & 0 & 0 \\
\hline Total & & 52 & $30(57,69 \%)$ & $9(17,3 \%)$ & $42(80,76 \%)$ & $2(3,84 \%)$ & $3(5,76 \%)$ \\
\hline
\end{tabular}

Pacheco \& Solari 1997) o fueron confrontados con la colección de Mamíferos del Departamento de Mastozoología del Museo de Historia Natural de la Universidad Nacional Mayor de San Marcos (UNMSM). También se realizó una colecta selectiva de plantas en floración o fructificación y se elaboraron catálogos referenciales de semillas y granos de polen. Las muestras botánicas fueron determinadas usando claves taxonómicas (Pennington et al. 2004, Brako \& Zarucchi 1993, Gentry 1993) y el apoyo de especialistas. Las determinaciones se corroboraron en el Herbario del Museo de Historia Natural de la UNMSM. El material fue depositado en la palinoteca del Laboratorio de Palinología y Paleobotánica y en el Herbario Magdalena Pavlich de la Universidad Peruana Cayetano Heredia.

Análisis de las muestras fecales.- Los análisis se realizaron sin conocer a que especie de murciélago pertenecía cada muestra. Primero se registraron semillas, tejidos vegetales y restos de insectos, mediante observaciones con estereoscopio. Las semillas registradas fueron determinadas utilizando la guía de Martin y Barkley (1961) y el catálogo referencial. Seguidamente, las muestras fueron sometidas al proceso de de acetólisis de Erdtman (1960) modificado y el material obtenido fue montado por duplicado con láminas portaobjetos y cubreobjetos, y fueron observadas al microscopio (40X). Los granos de polen registrados se determinaron con las claves taxonómicas de Roubik y Moreno (1991), Heusser (1971), Herrera y Urrego (1996) y el catálogo referencial. Finalmente los resultados se clasificaron en cuatro categorías: a) semillas, b) granos de polen, c) tejido vegetal y d) insectos. Se elaboró una lista de plantas consumidas en base a los resultados de semillas y granos de polen.

Índice Valor de Importancia de las Plantas (IVIP).- Se calculó utilizando las frecuencias de ocurrencia de granos de polen y semillas (planteado por Amaya 1991 citado en Muñoz-Saba et al. 1997). Entre mayor es el IVIP, mayor es el uso que una especie o comunidad de murciélagos da a una planta.

Análisis del nicho alimenticio.- En base a los resultados de granos de polen se estimó la amplitud de nicho alimenticio utilizando la medida de Levins estandarizada y la superposición de nicho alimenticio utilizando el índice de MacArthur y Levins modificado por Pianka (Krebs 1999). Estos índices fueron validados con la prueba estadística de Kruskal-Wallis (López \& Vaughan 2007). Además, sometimos los resultados de superposición de nicho a un análisis de agrupamiento. Para los análisis estadísticos se utilizó el programa PAST (Hammer et al. 2001).

\section{Resultados}

Análisis de las muestras fecales.- Se analizaron 52 muestras fecales pertenecientes a 9 especies de murciélagos de la familia Phyllostomidae: Carollia brevicauda, C. perspicillata, C. manu, Sturnira erythromos, S. oporaphilum, S. tildae, S. lilium, Anoura caudifer y $A$. geoffroyi. La mayoría de las muestras contenían tejidos vegetales $(80,7 \%, \mathrm{n}=42)$ y granos de polen $(57,6 \%, \mathrm{n}=$ 30). También se registraron 3 muestras estériles (Sánchez-Casas \& Álvarez 2000), es decir aquellas que no contenían ninguno de los 4 componentes analizados (Tabla 1).

En las muestras fecales se registraron 79 morfotipos de granos de polen y 9 morfoespecies de semillas. Los principales recursos consumidos pertenecieron a los géneros Piper (registrado en 23,08\% de las muestras), Cecropia (en 21,15\%) y Abutilon (en 13,46\%) (Apéndice 1). Las especies C. brevicauda, C. perspicillata, S. erythromos y $S$. oporaphilum consumieron en su mayoría especies de los géneros Piper y Cecropia, además S. erythromos consumió varias especies de la familia Solanaceae; mientras que A. caudifer consumió mayormente especies del género Abutilon. En las muestras fecales de C. manu, S. lilium y A. geoffroyi no se registraron los mencionados componentes.

Índice de Valor de Importancia de las plantas (IVIP).Los resultados del IVIP mostraron que para la comunidad de murciélagos evaluados en el valle de Kosñipata las plantas más importantes pertenecen a los géneros Piper, Cecropia y Abutilon, con $0,675,0,650$ y 0,385 de IVIP respectivamente (Tabla 2 ). Las plantas del género Piper son las más importantes para las especies $C$. brevicauda, C. perspicillata y $S$. erythromos, las plantas de Cecropia son las más importantes para $S$. oporaphilum, y las plantas de Abutilon para la especie $A$. caudifer.

Análisis del nicho alimenticio.- Los resultados de amplitud de nicho alimenticio según la medida de Levins estandarizada $\left(B_{A}\right)$ mostraron valores cercanos a cero para las 6 especies analizadas, indicando que son especialistas en su dieta en el área de estudio 
Tabla 2. Índice de Valor de Importancia de las plantas consumidas por los murciélagos. C. brevicauda (Cb), C. perspicillata (Cp), S. erythromos (Se), S. oporaphilum (So), S. tildae (St), A. caudifer (Ac), uso (P), índice de valor de importancia de las plantas (IVIP). * Sólo se muestran los resultados de IVIP mayores a 0,1 .

\begin{tabular}{|c|c|c|c|c|c|c|c|c|}
\hline \multirow{2}{*}{ Familia } & \multirow{2}{*}{ Género } & $\mathrm{Cb}$ & $\mathrm{Cp}$ & Se & So & St & Ac & \multirow{2}{*}{ IVIP* } \\
\hline & & $\mathbf{P}$ & $\mathbf{P}$ & $P$ & $\mathbf{P}$ & $\mathbf{P}$ & $\mathbf{P}$ & \\
\hline Piperaceae & Piper spp. & $\underline{0,154}$ & $\underline{0,200}$ & $\underline{0,250}$ & 0,071 & - & - & $\underline{0,675}$ \\
\hline Cecropiaceae & Cecropia spp. & 0,077 & 0,100 & 0,125 & $\underline{0,107}$ & 0,167 & 0,074 & $\underline{0,650}$ \\
\hline Malvaceae & Abutilon spp. & - & $\underline{0,200}$ & - & - & - & $\underline{0,185}$ & $\underline{0,385}$ \\
\hline Flacourtiaceae & Flacourtiaceae sp1. & 0,038 & - & 0,125 & - & - & 0,111 & 0,275 \\
\hline Melastomataceae & Miconia sp. & - & - & - & 0,036 & 0,167 & 0,037 & 0,239 \\
\hline Solanaceae & cf. Solanum sp. & - & - & - & - & 0,167 & 0,037 & 0,204 \\
\hline Euphorbiaceae & cf Omphalea sp. & - & - & - & 0,036 & 0,167 & - & 0,202 \\
\hline Asteraceae & Mikania sp. & - & - & 0,125 & 0,071 & - & - & 0,196 \\
\hline Rosaceae & Rubus spp. & 0,038 & 0,100 & - & - & - & 0,037 & 0,175 \\
\hline Moraceae-Urticaceae & Moraceae-Urticaceae sp4. & - & - & - & - & 0,167 & - & 0,167 \\
\hline Dicotiledonea & & - & - & - & - & 0,167 & - & 0,167 \\
\hline Cucurbitaceae & Cayaponia sp. & - & - & - & 0,036 & - & 0,111 & 0,147 \\
\hline Rubiaceae & Psychotria spp. & 0,038 & - & - & 0,071 & - & 0,037 & 0,147 \\
\hline Heliconiaceae & Heliconia sp. & 0,038 & 0,100 & - & - & - & - & 0,138 \\
\hline Bromeliaceae & Pitcairnia paniculata & - & 0,100 & - & - & - & 0,037 & 0,137 \\
\hline Bromeliaceae & Vriesea sp. & - & 0,100 & - & - & - & 0,037 & 0,137 \\
\hline Solanaceae & Solanum-Lycopersicum & 0,038 & - & 0,063 & 0,036 & - & - & 0,137 \\
\hline Solanaceae & Solanaceae sp2. & - & - & 0,125 & - & - & - & 0,125 \\
\hline Gentianaceae & cf. Chelonanthus sp. & - & - & - & 0,036 & - & 0,074 & 0,110 \\
\hline Ulmaceae & cf Celtis sp. & 0,038 & - & 0,063 & - & - & - & 0,101 \\
\hline Moraceae-Urticaceae & Moraceae-Urticaceae sp2. & - & 0,100 & - & - & - & - & 0,100 \\
\hline cf Ulmaceae & & - & 0,100 & - & - & - & - & 0,100 \\
\hline Indeterminada 6 & & - & 0,100 & - & - & - & - & 0,100 \\
\hline
\end{tabular}

(Tabla 3). La prueba de Kruskal-Wallis indicó que no existen diferencias significativas entre ellos ( $\mathrm{p}>0,05 ; \mathrm{H}=6,232, \mathrm{p}=0,397)$.

Los resultados del índice de MacArthur y Levins modificado por Pianka mostraron que existe una superposición casi completa entre la dieta de las especies C. brevicauda, C. perspicillata y $S$. erythromos (con 0,9999); mientras que los restantes niveles de superposición son todos menores a 0,5. La prueba de KruskalWallis indicó que existen diferencias significativas entre dichos resultados $(\mathrm{p}<0,05, \mathrm{H}=30,95, \mathrm{p}=0,005)$. El análisis de agrupamiento resultó en un dendrograma que agrupa a 3 de las especies analizadas, C. brevicauda, C. perspicillata y S. erythromos. $S$. oporaphilum muestra una dieta ligeramente similar al grupo anteriormente mencionado, mientras que $S$. tildae y $A$. caudifer presentan dietas disímiles a las demás especies (Fig. 1).

Tabla 3. Índice de Levins estandarizado $\left(B_{A}\right)$. Número de muestras $(\mathrm{N})$, medida de Levins (B).

\begin{tabular}{lccc}
\hline Especie & N & B & BA \\
\hline Carollia brevicauda & 6 & 1,1143 & 0,0014 \\
Carollia perspicillata & 2 & 1,0577 & 0,0007 \\
Sturnira erythromos & 8 & 1,0335 & 0,0004 \\
Sturnira oporaphilum & 6 & 1,9371 & 0,0119 \\
Sturnira tildae & 1 & 3,9032 & 0,0367 \\
Anoura caudifer & 5 & 4,5518 & 0,0450 \\
\hline
\end{tabular}

\section{Discusión}

Dieta.- La dieta de los murciélagos evaluados estuvo representada en su mayoría por las familias y géneros siguientes: Piperaceae (Piper), Cecropiaceae (Cecropia), Malvaceae (Abutilon) y Solanaceae. Todas ellas registradas como parte de la dieta de otras comunidades de murciélagos en el Neotrópico (Novoa et al. 2011, Arias et al. 2009, Lou \& Yurrita 2005, Thies \& Kalko 2004, López \& Vaughan 2004, Muchhala \& Jarrín-V 2002, Giannini 1999, Ascorra et al. 1996, Gorchov et al. 1995). Las

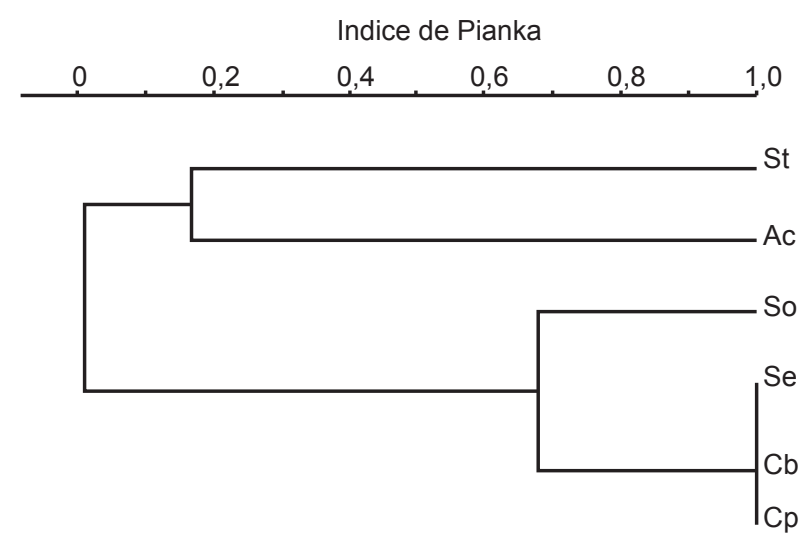

Figura 1. Análisis de agrupamiento. Abreviaciones: C. brevicauda (Cb), C. perspicillata (Cp), S. erythromos (Se), S. oporaphilum (So), S. tildae (St), A. caudifer (Ac). 
familias utilizadas para el consumo de frutos presentan frutos de tipo nuez o drupa sésiles en infrutescencias de tipo espiga (Cecropia y Piper) (Thies \& Kalko 2004, Gentry 1993), o de tipo baya como en el caso de Solanaceae (Gentry 1993). La familia Malvaceae, género Abutilon, que fue utilizada para el consumo de néctar-polen presenta algunas características que coinciden con las descritas para las plantas con síndrome de quiropterofilia (Muchhala \& Jarrín-V 2002), tales como, flores solitarias o agrupadas, con corola acampanada de colores claros, muchas veces en ramas terminales de fácil acceso para los murciélagos (Pennington et al. 2004).

Cada especie de murciélago mostró un mayor consumo hacia ciertos géneros o especies de plantas. Las especies del género Carollia mostraron un mayor consumo hacia plantas del género Piper. En las especies de Sturnira se observaron preferencias variadas, S. erythromos consumió mayormente plantas de Piper y varios taxa de la familia Solanaceae; mientras que $S$. oporaphilum consumió preferentemente plantas de Cecropia y Piper. En el caso de $A$. caudifer se observó un mayor consumo hacia plantas del género Abutilon. Estos resultados concuerdan con lo observado por otros autores para las especies de murciélagos evaluadas (Ortega \& Alarcon 2007, Lou \& Yurrita 2005, Thies \& Kalko 2004, López \& Vaughan 2004, Muchhala \& Jarrín-V 2002, Giannini 1999).

Además, los resultados obtenidos apoyan la idea de que los murciélagos frecuentemente tienden a consumir frutos de una o dos especies de plantas y complementan su dieta con otros recursos menos importantes para ellos (Lou \& Yurrita 2005, López 1996). Así como también, el registro del consumo de frutos de plantas pertenecientes a géneros o familias que contienen especies pioneras (Piper, Cecropia y la familia Solanaceae) (Thies \& Kalko 2004, Lobova et al. 2003, Olea-Wagner et al. 2007), apoya la importancia de los murciélagos frugívoros en los procesos de manutención de los bosques (Novoa et al. 2011, Fleming \& Heithaus 1981).

Las especies de los géneros Carollia y Sturnira analizadas, no son estrictamente frugívoras, ya que se ha registrado la ingesta de néctar-polen e insectos, aunque en menor cantidad que la ingesta de frutos (registrado por medio de tejidos vegetales y semillas). Del mismo modo, la especie $A$. caudifer tampoco es estrictamente nectarívora, debido al registro de tejidos vegetales en las muestras y a la determinación del uso de frutos de algunos taxa (Apéndice 1).

Índice de Valor de Importancia de las Plantas (IVIP): La importancia de los géneros Piper y Cecropia en la dieta de murciélagos frugívoros ha sido previamente recalcada por diversos autores (Lou \& Yurrita 2005, Gonçalves Da Silva et al. 2008, Mello et al. 2004, López \& Vaughan 2007, Thies \& Kalko 2004, Lobova et al. 2003, Giannini 1999), guardando concordancia con los resultados del IVIP (Tabla 2). Sin embargo, se contraponen a aquellos estudios que consideran a Cecropia como un complemento de la dieta de estos mamíferos (Mello et al. 2008, 2004, Muñoz-Saba et al. 1997).

La gran importancia del género Piper puede explicarse por el tipo de fenología que posee. Las inflorescencias contienen algunos frutos maduros en intervalos de tiempo predecibles durante largos periodos, fenología conocida como estado-estable; además presentan una maduración de frutos nocturna (Thies $\&$
Kalko 2004). En el caso del género Cecropia, puede deberse a su gran abundancia en la zona de estudio (obs. pers), ofreciendo considerables cantidades de frutos. Lo cual coincide con Dumont (2003), quien mencionó que Cecropia es relativamente abundante en las zonas tropicales y produce frutos constantemente.

El género Abutilon también ha sido mencionado como una fuente importante de néctar-polen para murciélagos nectarívoros neotropicales en los trabajos de Arias et al. (2009) y Sazima et al. (1999), lo cual concuerda con nuestros resultados. La importancia de estas plantas podría ser resultado del patrón de floración que presentan, como menciona Sazima et al. (1999) para la especie Abutilon aff. regnellii, presenta un patrón de floración continuo, ofreciendo néctar-polen a lo largo del año. Sin embargo, es necesario realizar estudios fenológicos de las especies de Abutilon de Kosńipata para tener certeza del patrón de floración que presentan.

Otra familia que es ampliamente mencionada en la literatura como utilizada por murciélagos del género Sturnira es Solanaceae (Giannini 1999, Mello et al. 2008, Lou \& Yurrita 2005, López \& Vaughan 2007), lo cual concuerda con nuestros resultados para la especie $S$. erythromos. Para los taxa de dicha familia se obtuvieron bajos IVIP, pero al calcular la sumatoria de los IVIP de los 4 taxa, el IVIP general para la familia Solanaceae pasa a ocupar el tercer lugar en la tabla de resultados con 0,528 ; lo cual realza la importancia de dicha familia en la dieta de los murciélagos del género Sturnira.

Análisis del nicho alimenticio.- El Índice de Levins estandarizado $\left(\mathrm{B}_{\mathrm{A}}\right)$ calculado indica que las especies C. brevicauda, $C$. perspicillata, S. erythromos, S. oporaphilum, $S$. tildae y $A$. caudifer son especialistas en su dieta en la zona de estudio. Los resultados de Lou y Yurrita (2005) sobre la amplitud de nicho alimenticio muestran que las especies C. brevicauda, C. perspicillata y $S$. lilium son bastante selectivas (especialistas). Igualmente, López y Vaughan (2007) obtuvieron bajos $\mathrm{B}_{\mathrm{A}}$ para 3 especies de Carollia. Sobre las especies del género Anoura, se menciona en trabajos previos, que pueden consumir una mayor variedad de recursos que las especies frugívoras (Caballero-Martínez et al. 2009, Arias et al. 2009), sin embargo no se han encontrado datos de amplitud de nicho alimenticio que indiquen si son especialistas o generalistas en sus dietas. Dentro del gremio de nectarívoros existen especies especialistas como Glossophaga soricina (Lou \& Yurrita 2005), por lo tanto no se descarta la posibilidad de que $A$. caudifer pueda tener un comportamiento selectivo al momento de consumir sus alimentos, pero es necesario estudios posteriores que analicen un mayor número de muestras fecales.

La prueba de Kruskal-Wallis para los resultados de $\mathrm{B}_{\mathrm{A}}$ indicó que no existen diferencias significativas entre ellos $(\mathrm{p}>0,05$; $\mathrm{H}=6,232, \mathrm{p}=0,397$ ), es decir, no hay una especie que sea más especialista en su dieta que otra en Kosñipata. Sin embargo, dada la variedad de registros en las muestras fecales de la especie $A$. caudifer, se considera que dicha especie sería menos especialista que las demás especies analizadas, pero para comprobar dicha afirmación es necesario el análisis de un mayor número de muestras.

Los resultados del índice de MacArthur y Levins modificado por Pianka mostraron que existe superposición entre las dietas de las especies C. brevicauda, C. perspicillata y S. erythromos (Tabla 4). Estos resultados coinciden con López (1996) (citado 
Tabla 4.- Índice de MacArthur y Levins modificado por Pianka. C. brevicauda (Cb), C. perspicillata (Cp), S. erythromos (Se), S. oporaphilum (So), S. tildae (St), A. caudifer (Ac).

\begin{tabular}{lcccccc}
\hline & Cb & Cp & Se & So & St & Ac \\
\hline C. brevicauda & - & 0,9999 & 0,9999 & 0,3528 & 0,0053 & 0,0016 \\
C. perspicillata & & - & 0,9999 & 0,3527 & 0,0040 & 0,0065 \\
S. erythromos & & & - & 0,3527 & 0,0009 & 0,0013 \\
S. oporaphilum & & & & - & 0,0143 & 0,0024 \\
S. tildae & & & & & - & 0,0850 \\
A. caudifer & & & & & & - \\
\hline
\end{tabular}

en Lou \& Yurrita 2005), López y Vaughan (2007) y Lou y Yurrita (2005), quienes mencionan que existen altos niveles de superposición entre las dietas de especies de Carollia, Sturnira y otros Stenodermatinos. Morfológicamente las especies de Carollia y Sturnira son de tallas similares, lo cual origina mayor superposición de nicho trófico, ya que los frutos que consumen por lo general son de tamańos similares (Lou \& Yurrita 2005).

En conclusión, se ha logrado documentar las interacciones que existen entre los murciélagos y las plantas en el valle de Kosñipata, evidenciadas por el uso que los murciélagos hacen de los frutos y el néctar-polen como parte de su dieta. Cabe resaltar que estos murciélagos podrían estar desempeñando importantes roles de dispersión y polinización, siendo entes fundamentales en los procesos de sucesión y manutención del bosque.

\section{Agradecimientos}

Nuestro especial agradecimiento al Blgo. Daniel Blanco y a los trabajadores del albergue turístico "Cock of the rocks". Al PhD. Víctor Pacheco, Director del Departamento de Mastozoología del Museo de Historia Natural (UNMSM), por la revisión y correcciones al presente trabajo. A Carla Delgado, Alessandra Quiñones, Cindy Vergel, Aaron Cánepa, Jorge León, Daniel Ramos y Alfredo Butrón, por su participación en la colecta de muestras en Kosñipata.

\section{Literatura citada}

Albuja L. 1999. Quirópteros del Ecuador. Editorial Cicetronic Cia. Ltda. Offset. Quito-Ecuador; 2da edición.

Angulo S.R, Escobedo M, López C \& Ríos J.A. 2004. Introducción al estudio y conservación de los Quirópteros Neotropicales. Universidad Nacional de la Amazonía Peruana. Iquitos, Perú.

Arias E., Cadenillas R., Pacheco V. 2009. Dieta de murciélagos nectarívoros del Parque Nacional Cerros de Amotape, Tumbes. Rev. peru. biol. 16(2): $187-190$.

Ascorra C.F., Solari S. \& Wilson D. E. 1996. Diversidad y ecología de los quirópteros en Pakitza. Pp. 585-604. En "Manu: the Biodiversity of southeastern Peru" (d.E. Wilson \& A. Sandoval, eds.). Smithsonian Institution, Washington, DC.

Brako L. \& Zarucchi J. L. 1993. Catálogo de las angiospermas y gimnospermas del Perú. Monographs in Sistematic Botany. Missouri Botanical Garden. Vol. 45.

Caballero-Martinez L. A., Rivas I. V. \& Aguilera L. I. 2009. Hábitos alimentarios de Anoura geoffroyi (Chiroptera: Phyllostomidae) en Ixtapan del Oro, Estado de México. Acta Zoológica Mexica (n. s.) 25(1): $161-175$.

Delgado C. 2010. Variación estacional en la diversidad y estado reproductivo de quirópteros con énfasis en los géneros Sturnira y Carollia (Chiroptera: Phyllostomidae) en el bosque nublado de San Pedro, Cusco (Tesis). Universidad Peruana Cayetano Heredia.
Dumont E. R. 2003. Bats and fruit: an ecomorphological approach. En T. H. Kunz and M. B. Fenton (Eds). Bat Ecology, Pp. 398-421. The University of Chicago Press, Chicago, Illinois.

Erdtman G. 1960. The acetolysis method. Svensk. Bot Tidshkr. 54(4): $561-564$.

Fleming T. H. 1988. The short-tailed fruit bat: a study in plantanimal interactions. University of Chicago Press, Chicago, Illinois, USA.

Fleming T. H. \& Heithaus E. R. 1981. Fugivorous bats, seed shadows, and the structure of Tropical Forests. Biotropica, Vol. 13, No. 2, Supplement: Reproductive Botany $45-43$.

Gardner A. L. (Ed.) 2007. Mammals of South America. Volume 1: Marsupials, Xenarthrans, Shrews and Bats. USA.

Gentry A. 1993. A field guide to the families and genera of Woody Plants of Northwest South America (Colombia, Ecuador, Peru) with supplementary notes on herbaceous taxa. Conservation International. Washington, DC. USA.

Giannini N. P. 1999. Selection of diet and elevation by sympatric species os Sturnira in an Andean Rainforest. Journal of Mammology 80(4): 1186 - 1195.

Gonçalves Da Silva A., Gaona A. \& Medellin R. 2008. Diet and Trophic structure in a community of fruit-eating bats in Lacandon Forest, Mexico. Journal of Mammology 89(1): 43 - 49.

Gorchov D. L., Cornejo F., Ascorra C. F. \& JARAMILLO M. 1995. Dietary overlap between frugivorous birds and bats in the Peruvian Amazon. Oikos 74: 235 - 250.

Hammer Ø, Harper, D.A. \& RYAN P.D. 2001. PAST: Paleontological Statistics Software Package for Education and Data Analysis. Paleontologia Electronica 4(1).

Herrera L. F. \& Urrego L. E. 1996. Atlas de polen de plantas útiles y cultivadas de la Amazonía colombiana. Volumen XL. Tropenbos. Colombia.

Heusser C. 1971. Pollen and spores of Chile. The University of Arizona Press. United States of America.

Holdrige L. 1978. Ecología Basada en Zonas de Vida. IICA. San José - Costa Rica.

Krebs C. J. 1999. Ecological Methodology. Second Edition. AddisonWesley Educational Publishers, Inc. Benjamin/Cummings. Menlo Park, California.

Lobova T. A., Mori S., Blanchard F., Peckham H. \& CharlesDominique P. 2003. Cecropia as a food resource for bats in French Guiana and the significance of fruit structure in seed dispersal and longevity. American Journal of Botany 90(3): 388-403.

Loayza P., Rios R. \& Larrea-Alcazar D. 2006. Disponibilidad de recurso y dieta de murciélagos frugívoros en la Estación Biológica de Tunquini, Bolivia. Ecología en Bolivia 41(1): 7-23.

Lopez J. E., Vaughan C. 2004. Observations on the role of frugivorous bats as seed dispersers in Costa Rican secondary humid forests. Acta Chiropterologica 6(1): $111-119$.

Lopez J. E. \& Vaughan C. 2007. Food Niche Overlap Among Neotropical Frugivorous bats in Costa Rica. Rev. Biol. Trop. Vol.55(1):301-313.

Lou S. \& Yurrita C. 2005. Análisis de nicho alimentario en la comunidad de murciélagos frugívoros de Yaxhá, Petén, Guatemala. Acta Zoológica Mexicana (n. s.) 21(1): 83 - 94.

Martin A. C. \& Barkley W. D. 1961. Seed Identification Manual. University of California Press. Berkely and Los Angeles, USA.

Mello M. A., Schittini G. M., Selig P. \& Bergallo H. G. 2004. Seasonal variation in the diet of the bat Carollia perspicillata (Chiroptera: Phyllostomidae) in an Atlantic Forest area in southeastern Brazil. Mammalia 68(1): 49-55.

Mello M., Kalko E. \& Silva W. 2008. Diet and abundance of the bat Sturnira lilium (Chiroptera) in a Brazilian Montane Atlantic Forest. Journal of Mammalogy 89(2): 485-492. 
Muchhala N. \& Jarrin-V P. 2002. Flower visitation by bats in cloud forests of western Ecuador. Biotropica 34(3): 387-395.

Muñoz-Saba Y., Cadena A. \& Rangel-CH J. 1997. Ecología de los murciélagos antófilos del sector La Curia, Serrania La Macarena, Colombia. Rev. Acad. Colomb. Cienc. 21(81):473-486.

Novoa S., Cadenillas R. \& Pacheco V. 2011. Dispersión de semillas por murciélagos frugívoros en bosques del Parque Nacional Cerros de Amotape, Tumbes, Perú. Mastozoología Neotropical 18(1): 81-93.

Olea-Wagner A., Lorenzo C., Naranjo E., Ortiz D. \& Leon-Paniagua L. 2007. Diversidad de frutos que consumen tres especies de murciélagos (Chiroptera:Phyllostomidae) en la selva lacandona, Chiapas, México. Revista Mexicana de Biodiversidad 78: 191-200.

Ortega J. \& Alarcon I. 2007. Anoura geoffroyi (Chiroptera: Phyllostomidae). Mammalia Species 818: 1-7.

Pacheco V. \& Solari S. 1997. Manual de los Murciélagos Peruanos con énfasis en las especies hematófagas. Organización Panamericana de la Salud. 74 pp.
Pennington T. D., Reynel C. \& Daza A. 2004. Illustrated guide to the Trees of peru. David Hunt, The Manse, Chapel Lane, Milborne Port Sherborne, DT9 5DL. England.

Roubik D. W. \& Moreno J. E. 1991. Pollen and spores of Barro Colorado Island. Missouri Botanical Garden (Eds.). USA.

Sanchez-Casas N. \& Alvarez T. 2000. Palinofagia de los murciélagos del género Glossophaga (Mammalia: Chiroptera) en México. Acta Zool. Mex. (n. s.) 81: 23-62.

Sazima M., Buzato S. \& Sazima I. 1999. Bat-pollinated flower assemblages and bat visitors at two Atlantic Forest sites in Brazil. Annals of Botany 83: 705-712.

SENAMHI Servicio Nacional de Meteorología e Hidrología del Perú. 2010. (en línea). <http://www.senamhi.gob.pe/main_mapa. php? $\mathrm{t}=\mathrm{dHi}>$. Acceso 02/2010.

Smith N., Mori S. A., Henderson A., Stevenson D. W. \& Heald S. V. 2004. Flowering plants of the Neotropics. New York Botanical Garden. Princeton University Press. Italia.

Thies W. \& Kalko E. K. V. 2004. Phenology of neotropical pepper plants (Piperaceae) and their association with their main dispersers, two short-tailed fruit bats, Carollia perspicillata and C. castanea (Phyllostomidae). Oikos 104: 362-376.

Apéndice 1. Listado de plantas registradas en las muestras fecales de los murciélagos del valle de Kosñipata, indicando sus formas de uso y las variables frecuencia y abundancia. Frutos (f), néctar-polen (p), indefinido (i), C. brevicauda (Cb), C. perspicillata (Cp), S. erythromos (Se), S. oporaphilum (So), S. tildae (St), A. caudifer (Ac), número de muestras (n), frecuencia (F), abundancia (A).

\begin{tabular}{|c|c|c|c|c|c|c|c|c|c|c|c|c|c|c|}
\hline \multirow{2}{*}{ Familia } & \multirow{2}{*}{ Especie } & \multirow{2}{*}{ Uso } & \multicolumn{2}{|c|}{$\mathrm{Cb}$} & \multicolumn{2}{|c|}{$\mathrm{Cp}$} & \multicolumn{2}{|c|}{ Se } & \multicolumn{2}{|c|}{ So } & \multicolumn{2}{|c|}{ St } & \multicolumn{2}{|c|}{ Ac } \\
\hline & & & $\mathbf{F}$ & $\mathbf{A}$ & $\mathbf{F}$ & $\mathbf{A}$ & $\mathbf{F}$ & $\mathbf{A}$ & $\mathbf{F}$ & $\mathbf{A}$ & $\mathbf{F}$ & $\mathbf{A}$ & $\mathbf{F}$ & $\mathbf{A}$ \\
\hline \multicolumn{15}{|l|}{ SEMILLAS } \\
\hline Cecropiaceae & Cecropia latiloba & $\mathrm{f}$ & & & & & 1 & 3 & & & & & & \\
\hline Clusiaceae & Vismia tomentosa & $\mathrm{f}$ & & & & & 1 & 1 & & & & & & \\
\hline \multirow{4}{*}{ Piperaceae } & Piper cf crassinervium & $\mathrm{f}$ & 1 & 2 & 1 & 5 & & & & & & & & \\
\hline & Piper sp1. & $\mathrm{f}$ & & & 1 & 1 & & & & & & & & \\
\hline & Piper sp2. & $\mathrm{f}$ & & & & & 1 & 25 & & & & & & \\
\hline & Piper sp3. & $\mathrm{f}$ & & & & & 1 & 3 & & & & & & \\
\hline Solanaceae & Solanum appressum & $\mathrm{f}$ & & & & & & & & & 1 & 2 & & \\
\hline cf. Fabaceae & & $\mathrm{f}$ & & & & & & & 1 & 1 & & & & \\
\hline cf. Moraceae & & $\mathrm{f}$ & & & & & & & 1 & 1 & & & & \\
\hline
\end{tabular}

GRANOS DE POLEN

\begin{tabular}{|c|c|c|c|c|c|c|c|c|c|c|}
\hline \multirow{3}{*}{ Araceae } & Dieffenbachia sp. & $\mathrm{f}$ & 1 & 3 & & & & & & \\
\hline & Araceae sp1. & $\mathrm{f}$ & 1 & 1 & & & & & & \\
\hline & Araceae sp2. & $\mathrm{f}$ & 1 & 3 & & & & & & \\
\hline \multirow{3}{*}{ Arecaceae } & Arecaceae sp1. & $\mathrm{f}$ & 1 & 2 & & & & & & \\
\hline & Arecaceae sp2. & $\mathrm{f}$ & & & 1 & 2 & & & & \\
\hline & Arecaceae sp3. & $\mathrm{f}$ & & & & & 1 & 2 & & \\
\hline \multirow{2}{*}{ Asteraceae } & Mikania sp. & $\mathrm{p}$ & & & 2 & 2 & 2 & 3 & & \\
\hline & Asteraceae sp1. & $\mathrm{p}$ & & & & & 1 & 1 & & \\
\hline \multirow{5}{*}{ Bignoniaceae } & cf. Tynanthus sp. & $\mathrm{p}$ & & & & & & & 1 & 211 \\
\hline & cf. Callychlamys sp. & $\mathrm{p}$ & & & & & & & 1 & 4 \\
\hline & Bignoniaceae sp1. & $\mathrm{p}$ & & & & & 1 & 20 & & \\
\hline & Bignoniaceae sp2. & $\mathrm{p}$ & & & & & & & 1 & 1 \\
\hline & Bignoniaceae sp3. & $\mathrm{p}$ & & & & & & & 1 & 52 \\
\hline
\end{tabular}


Apéndice 1. Continuación.

\begin{tabular}{|c|c|c|c|c|c|c|c|c|c|c|c|c|c|c|}
\hline \multirow{2}{*}{ Familia } & \multirow{2}{*}{ Especie } & \multirow{2}{*}{ Uso } & \multicolumn{2}{|c|}{$\mathrm{Cb}$} & \multicolumn{2}{|c|}{$\mathrm{Cp}$} & \multicolumn{2}{|c|}{ Se } & \multicolumn{2}{|c|}{ So } & \multicolumn{2}{|c|}{ St } & \multicolumn{2}{|c|}{ Ac } \\
\hline & & & $F$ & $\mathbf{A}$ & $\mathbf{F}$ & A & $\mathbf{F}$ & $\mathbf{A}$ & $\mathbf{F}$ & A & F & A & F & A \\
\hline \multirow{3}{*}{ Bombacaceae } & Ochroma sp. & $\mathrm{p}$ & & & & & & & & & & & 2 & 6 \\
\hline & cf. Matisia sp. & $\mathrm{p}$ & & & & & & & & & & & 1 & 4 \\
\hline & Bombacaceae sp1. & $\mathrm{p}$ & & & & & 1 & 1 & & & & & & \\
\hline \multirow{4}{*}{ Bromeliaceae } & Pitcairnia paniculata & $\mathrm{p}$ & & & 1 & 4 & & & & & & & 1 & 5661 \\
\hline & Vriesea sp. & $\mathrm{p}$ & & & 1 & 3 & & & & & & & 1 & 12 \\
\hline & cf. Billbergia sp. & $\mathrm{f}$ & & & & & 1 & 2 & & & & & & \\
\hline & Bromeliaceae sp1. & $\mathrm{f}, \mathrm{p}$ & & & & & & & 2 & 3 & & & & \\
\hline Burseraceae & cf. Protium sp. & $\mathrm{p}$ & & & & & & & & & & & 1 & 14 \\
\hline Cecropiaceae & Cecropia spp. & $\mathrm{f}, \mathrm{p}$ & 2 & 12 & 1 & 7 & 1 & 2 & 3 & 80 & 1 & 4 & 2 & 1037 \\
\hline Clusiaceae & cf Vismia sp. & $\mathrm{f}$ & 1 & 1 & & & & & & & & & & \\
\hline \multirow{2}{*}{ Cucurbitaceae } & Cayaponia sp. & $\mathrm{p}$ & & & & & & & 1 & 5 & & & 3 & 199 \\
\hline & Gurania sp. & $\mathrm{f}$ & & & & & & & & & & & 1 & 1 \\
\hline Cyclanthaceae & cf Asplundia sp. & $\mathrm{f}$ & & & & & & & 1 & 1 & & & & \\
\hline Ericaceae & Cavendishia sp. & $\mathrm{f}$ & & & & & & & & & & & 1 & 1 \\
\hline \multirow{4}{*}{ Euphorbiaceae } & Acalypha cf macrostachya & $\mathrm{p}$ & & & & & & & & & & & 1 & 7 \\
\hline & cf Alchronea sp. & $\mathrm{p}$ & & & & & & & 1 & 5 & & & & \\
\hline & cf Omphalea sp. & $\mathrm{f}, \mathrm{p}$ & & & & & & & 1 & 2 & 1 & 1 & & \\
\hline & cf Sapium sp. & $\mathrm{f}$ & & & & & & & 1 & 3 & & & & \\
\hline \multirow{5}{*}{ Fabaceae } & Inga sp. & $\mathrm{p}$ & & & & & & & 1 & 14 & & & & \\
\hline & cf Dioclea sp. & i & & & & & 1 & 1 & & & & & & \\
\hline & cf Mucuna sp. & $\mathrm{p}$ & & & & & & & & & & & 1 & 5 \\
\hline & Mimosoidea & $\mathrm{p}$ & & & & & & & 1 & 4 & & & & \\
\hline & Caesalpinoidea & $\mathrm{p}$ & 1 & 11 & & & & & & & & & & \\
\hline Flacourtiaceae & Flacourtiaceae sp1. & $\mathrm{f}, \mathrm{p}$ & 1 & 3 & & & 2 & 5 & & & & & 3 & 3243 \\
\hline Gentianaceae & cf. Chelonanthus sp. & $\mathrm{p}$ & & & & & & & 1 & 48 & & & 2 & 23 \\
\hline Gesneriaceae & Gesneriaceae sp1. & $\mathrm{f}, \mathrm{p}$ & 1 & 3 & & & & & 1 & 4 & & & & \\
\hline Heliconiaceae & Heliconia sp. & $\mathrm{p}$ & 1 & 1 & 1 & 6 & & & & & & & & \\
\hline Malvaceae & Abutilon spp. & $\mathrm{p}$ & & & 2 & 10 & & & & & & & 5 & 4249 \\
\hline Melastomataceae & Miconia sp. & $\mathrm{f}, \mathrm{p}$ & & & & & & & 1 & 2 & 1 & 3 & 1 & 4 \\
\hline \multirow{2}{*}{ Moraceae } & Ficus sp. & $\mathrm{f}$ & & & & & & & 1 & 1 & & & & \\
\hline & Sorocea sp. & $\mathrm{i}$ & & & & & & & 1 & 4 & & & & \\
\hline \multirow{5}{*}{$\begin{array}{l}\text { Moraceae- } \\
\text { Urticaceae }\end{array}$} & Moraceae-Urticaceae sp1. & $\mathrm{i}$ & 1 & 1 & & & & & & & & & & \\
\hline & Moraceae-Urticaceae sp2. & $\mathrm{i}$ & & & 1 & 2 & & & & & & & & \\
\hline & Moraceae-Urticaceae sp3. & $\mathrm{i}$ & & & & & 1 & 1 & & & & & & \\
\hline & Moraceae-Urticaceae sp4. & $\mathrm{i}$ & & & & & & & & & 1 & 2 & & \\
\hline & Moraceae-Urticaceae sp5. & i & & & & & & & & & & & 1 & 23 \\
\hline Piperaceae & Piper spp. & $\mathrm{f}$ & 4 & 1615 & 2 & 1262 & 4 & 1564 & 2 & 1450 & & & & \\
\hline \multirow{2}{*}{ Rosaceae } & Rubus cf urticiifolius & $\mathrm{f}$ & & & & & & & 1 & 1 & & & & \\
\hline & Rubus spp. & $\mathrm{f}, \mathrm{p}$ & 1 & 2 & 1 & 2 & & & & & & & 1 & 9 \\
\hline \multirow{6}{*}{ Rubiaceae } & Genipa sp. & $\mathrm{p}$ & & & & & & & & & & & 1 & 3936 \\
\hline & Nertera sp. & $\mathrm{f}, \mathrm{p}$ & & & & & 1 & 3 & & & & & & \\
\hline & Psychotria spp. & f, p & 1 & 2 & & & & & 2 & 2 & & & 1 & 1 \\
\hline & cf Warsewiczia sp. & $\mathrm{p}$ & 1 & 13 & & & & & & & & & & \\
\hline & Rubiaceae sp1. & $\mathrm{f}, \mathrm{p}$ & 1 & 2 & & & & & & & & & 1 & 1 \\
\hline & Rubiaceae sp2. & $\mathrm{f}, \mathrm{p}$ & & & & & & & & & & & 1 & 3 \\
\hline
\end{tabular}


Maguiña

Apéndice 1. Continuación.

\begin{tabular}{|c|c|c|c|c|c|c|c|c|c|c|c|c|c|c|}
\hline \multirow{2}{*}{ Familia } & \multirow{2}{*}{ Especie } & \multirow{2}{*}{ Uso } & \multicolumn{2}{|c|}{$\mathrm{Cb}$} & \multicolumn{2}{|c|}{$\mathrm{Cp}$} & \multicolumn{2}{|c|}{ Se } & \multicolumn{2}{|c|}{ So } & \multicolumn{2}{|c|}{ St } & \multicolumn{2}{|c|}{ Ac } \\
\hline & & & $\mathbf{F}$ & $\mathbf{A}$ & $\mathbf{F}$ & $\mathbf{A}$ & $\mathbf{F}$ & A & $\mathbf{F}$ & A & $\mathbf{F}$ & A & $\mathbf{F}$ & $\mathbf{A}$ \\
\hline \multirow{4}{*}{ Solanaceae } & cf. Solanum sp. & $\mathrm{f}$ & & & & & & & & & & & 1 & 1 \\
\hline & Solanum-Lycopersicum & $\mathrm{f}, \mathrm{p}$ & 1 & 1 & & & 1 & 2 & 1 & 225 & & & & \\
\hline & Solanaceae sp1. & $\mathrm{f}$ & & & & & 1 & 1 & & & & & & \\
\hline & Solanaceae sp2. & $\mathrm{f}$ & & & & & 2 & 3 & & & & & & \\
\hline \multirow{2}{*}{ Ulmaceae } & cf Celtis sp. & $\mathrm{i}$ & 1 & 1 & & & 1 & 1 & & & & & & \\
\hline & Trema micrantha & $\mathrm{i}$ & 1 & 2 & & & & & & & & & & \\
\hline cf. Bombacaceae & & $\mathrm{p}$ & & & & & & & 1 & 3840 & & & & \\
\hline cf. Fabaceae & & $\mathrm{p}$ & & & & & & & & & & & 1 & 58 \\
\hline cf. Solanaceae & & $\mathrm{f}$ & 1 & 9 & & & & & & & & & & \\
\hline cf. Ulmaceae & & $\mathrm{i}$ & & & 1 & 1 & & & & & & & & \\
\hline Dicotiledonea & & $\mathrm{i}$ & & & & & & & & & 1 & 1 & & \\
\hline Indeterminada 1 & & $\mathrm{i}$ & 1 & 7 & & & & & & & & & & \\
\hline Indeterminada 2 & & $\mathrm{i}$ & 1 & 1 & & & & & & & & & & \\
\hline Indeterminada 3 & & $\mathrm{i}$ & 1 & 1 & & & & & & & & & & \\
\hline Indeterminada 4 & & $\mathrm{i}$ & 1 & 1 & & & & & & & & & & \\
\hline Indeterminada 5 & & $\mathrm{i}$ & 1 & 1 & & & & & & & & & & \\
\hline Indeterminada 6 & & $\mathrm{i}$ & & & 1 & 1 & & & & & & & & \\
\hline Indeterminada 7 & & i & & & & & 1 & 1 & & & & & & \\
\hline Indeterminada 8 & & $\mathrm{i}$ & & & & & & & 1 & 1 & & & & \\
\hline Indeterminada 9 & & $\mathrm{i}$ & & & & & & & 1 & 1 & & & & \\
\hline Indeterminada 10 & & $\mathrm{i}$ & 1 & 1 & & & & & 1 & 1 & & & & \\
\hline
\end{tabular}

